\title{
Electrochemistry of a Thin Cobalt(II)-Heptacyanonitrosylferrate Film Modified Glassy Carbon Electrode
}

\author{
Zhiqiang GAO*广, Yimin ZHANG** and Guangqing WANG*** \\ * Department of Chemistry, National University of Singapore, Kent Ridge Crescent, \\ Singapore 119260, Republic of Singapore \\ **Laboratory of Electronic Microscope, Luoyang Technological Institute, Luoyang, \\ Henan, People's Republic of China \\ ***Department of Chemistry, Yulin Normal Collage, Yulin, Shanxi, People's Republic of China
}

\begin{abstract}
The electrochemical behavior of a cobalt(II)-heptacyanonitrosylferrate (CoHNF) film on a glassy carbon electrode has been investigated. Cyclic voltammetry shows a single electron-transfer reaction taking place at $0.51 \mathrm{~V}$ for the modified electrode. The ion effect of the supporting electrolyte indicates that the ion permeability in the CoHNF film is mainly determined by the hydrated radius of cation. The modified electrode catalyzes redox reactions of the $\mathrm{Fe}^{2+} / \mathrm{Fe}^{3+}$ couple. It also exhibits a linear potentiometric response over the range of $3 \times 10^{-5}-1 \times 10^{-2} \mathrm{M} \mathrm{NO}_{2}^{-}$with a near-Nernstian slope in an acidic medium.
\end{abstract}

Keywords Cobalt, heptacyanonitrosylferrate, chemically modified electrode, voltammetry, electrochemical impedance, nitrite, iron

During the past years inorganic film modified electrodes have received increased attention due to their potentials in technical applications. Various inorganic materials, such as clays ${ }^{1}$, zeolites $^{2}$, metal $_{\text {oxides }}^{3}$, metal phthalocyanines ${ }^{4}$, metal porphyrins ${ }^{5}$, transition metal ${ }^{6}$, polyoxometallates ${ }^{7}$ and polynuclear transition metal cyanides $^{8-10}$, have been used to fabricate inorganic film modified electrodes. Of these, the use of polynuclear transition metal cyanides as modifiers appears to be especially attractive because a large number of these compounds can be easily prepared. In addition, the well-defined zeolite-like structure makes the description and understanding of the charge-transfer process easier compared with that of organic polymer films. ${ }^{11}$

Since the pioneering work of Neff and Itaya ${ }^{8}$ on Prussian Blue modified electrodes, the field of polynuclear transition metal cyanides modified electrodes has undergone considerable progress over the past years. However, considering the limited diversity of these chemically synthesized compounds, there is still muchwork to be done.

In this paper, we report on a method for preparing thin films of CoHNF on glassy carbon electrode surface. The modified electrode exhibits excellent stability in aqueous media. The parameters which affect the voltammetric behavior were examined. Possible analytical applications were attempted.

\footnotetext{
$\doteqdot$ To whom correspondence should be addressed.
}

\section{Experimental}

\section{Materials and apparatus}

All chemicals were of certified analytical grade (Merck) and used as received. Solutions were prepared with distilled deionized water from a Millipore system (Millipore, Milford, MA, USA). A 3-mm diameter glassy carbon electrode was polished and cleaned according to a procedure published elsewhere. ${ }^{10}$

Cyclic voltammetric and electrochemical impedance spectroscopic (EIS) experiments of the modified electrode were carried out as described previously. ${ }^{10}$ All electrochemical measurements were conducted using a conventional three-electrode system with a triangle arrangement. The modified electrode was used as the working electrode, with a platinum foil counter electrode and a saturated calomel reference electrode (SCE). All potentials reported in this work are referred to the the SCE.

\section{Procedures}

The glassy carbon electrode was scanned from -0.6 to $-1.15 \mathrm{~V}$ in an oxygen-free $1 \times 10^{-2} \mathrm{M} \mathrm{CoCl}_{2}-0.1 \mathrm{M}$ $\mathrm{KNO}_{3}$ solution with a potential scan rate of $20 \mathrm{mV} / \mathrm{s}$ and kept at a potential of $-1.15 \mathrm{~V}$ for a certain period of time. After being removed from the solution and thoroughly rinsed with water, the electrode was dipped in a $0.1 \mathrm{M} \mathrm{K}_{2}\left[\mathrm{Fe}(\mathrm{CN})_{5} \mathrm{NO}\right]$ solution and treated by potential cycling between -0.2 and $0.75 \mathrm{~V}$ until a steady state voltammogram was observed. Thick CoHNF films 
could be deposited on the glassy carbon electrode by repeating the above procedure.

\section{Results and Discussion}

\section{Voltammetry of the CoHNF film modified electrode}

Figure 1 shows steady-state cyclic voltammograms of the modified electrode. For the first few cycles, a pronounced tailing of the $i-E$ curve was observed. With increasing the number of cyclic scans, this tailing gradually disappeared and a well-defined steady-state $i-E$ curve was obtained. This behavior of the fresh modified electrode may be due to the migration of free $\mathrm{Co}^{2+}$ from the lattice of the film to the solution. Upon potential cycling, the $\mathrm{Co}^{2+}$ ions were replaced by $\mathrm{K}^{+}$ions, resulting in a typical steady state voltammogram.

As illustrated in Fig. 1, the modified electrode exhibited exactly as expected for a highly reversible surfaceimmobilized redox couple. The peak currents at $0.51 \mathrm{~V}$ were found to be linear with the potential scan rate up to $200 \mathrm{mV} / \mathrm{s}$, and the ratio of the anodic to the cathodic charge obtained by integrating the $i-E$ curve at a very slow potential scan rate was close to unity. The derivation from linearity accompanying the observable tailing current occurred when increasing the film thickness. The voltammogram was almost symmetrical at low potential scan rates, and the peak-to-peak potential separation $\left(\Delta E_{\mathrm{p}}\right)$ was less than $20 \mathrm{mV}$, whereas $\Delta E_{\mathrm{p}}$ increased with increasing potential scan rate and film thickness.

The crystal structure of CoHNF is a face-centered cubic lattice with a cell constant of $10.2-10.6 \AA .^{12}$ The redox process would require ion transportation for charge balance. Thus, ions in the electrolyte solution

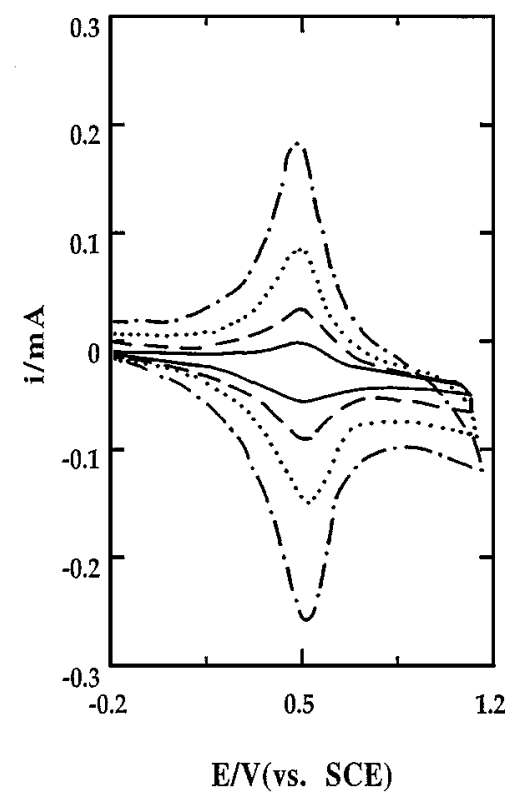

Fig. 1 Cyclic voltammograms of the CoHNF modified electrode in $1.0 \mathrm{M} \mathrm{KCl}$. Potential scan rates of (-) 20, (--) 50, ( ... $) 100$ and (-.-) $200 \mathrm{mV} / \mathrm{s}$. must have a profound effect on the electrochemical behavior of the modified electrode. In a solution of $\mathrm{K}^{+}$, $\mathrm{Na}^{+}, \mathrm{Li}^{+}$and $\mathrm{NH}_{4}{ }^{+}$, the cyclic voltammograms were well defined, indicating that these ions can transport through the film freely. In the presence of $\mathrm{Ba}^{2+}, \mathrm{Mg}^{2+}$ and $\mathrm{Ca}^{2+}$, the typical voltammetric response of the film disappeared after dozens of cycles (Fig. 2). However, when a $1 \mathrm{M} \mathrm{KCl}-1 \mathrm{M} \mathrm{CaCl}_{2}$ solution was employed, the shape of the voltammogram was essentially the same as that in the $1 \mathrm{M} \mathrm{KCl}$ solution. Furthermore the electrochemical properties of the modified electrode were only little affected by the nature of the anion present in the electrolyte solution. The selective ion transport can be readily explained in terms of hydrated ionic radii and the channel radius in the CoHNF lattice, as been in the case of Prussian-Blue film modified electrodes. ${ }^{8}$ The electrostatic factor, ionic polarizability and structural disorder of the microcrystalline deposit can also affect ion transportation through the film. The stability of the film was in fairly good coincidence with the effect of the ion on the film, the smaller radius of the cation, easier transport through the film; also, higher stability and larger peak currents were observed.

The effect of the electrolyte concentration on the voltammetric behavior is illustrated in Fig. 3. It can be

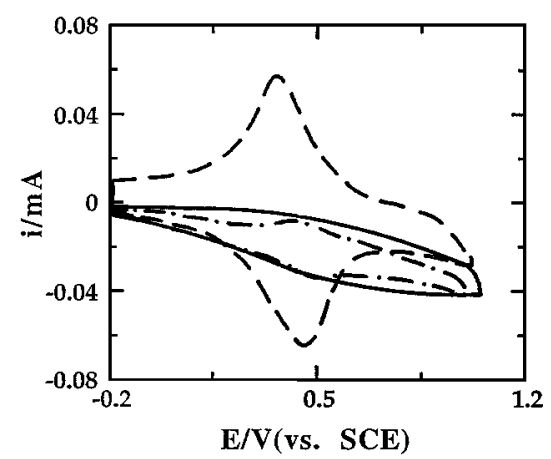

Fig. 2 Cyclic voltammograms (the 20th cycle) of the CoHNF modified electrode in $1.0 \mathrm{M} \mathrm{CaCl}_{2}(-), \mathrm{BaCl}_{2}(-\cdot-)$ and 1.0 $\mathrm{M} \mathrm{LiCl}(\ldots)$. Potential scan rate $100 \mathrm{mV} / \mathrm{s}$.

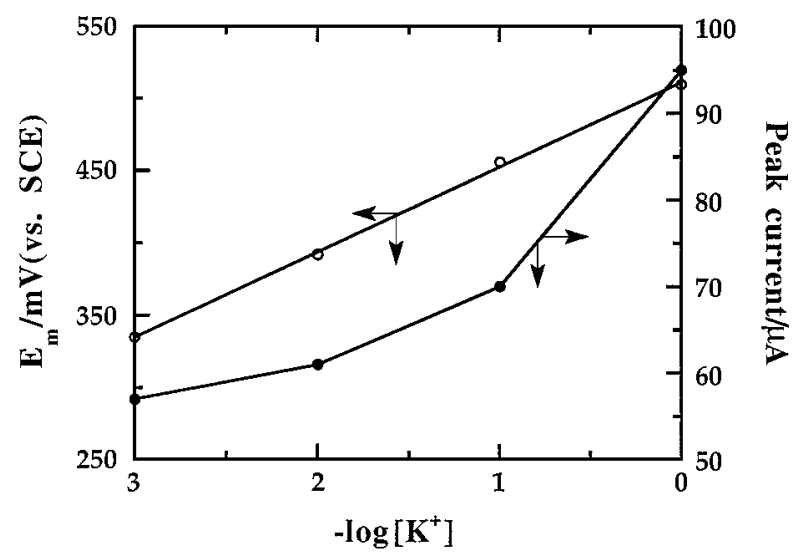

Fig. 3 Dependence of the $E_{\mathrm{m}}$ and peak current on the concentration of $\mathrm{KCl}$. The potential scan rate is $100 \mathrm{mV} / \mathrm{s}$. 
seen that $E_{\mathrm{m}}\left[E_{\mathrm{m}}=1 / 2\left(E_{\mathrm{pa}}+E_{\mathrm{pc}}\right)\right]$ shifted anodically with increasing $\mathrm{KCl}$ concentration. Good linearity was observed in the concentration range of $1.0 \mathrm{mM}$ to 1.0 M. The peak currents decreased upon lowering the electrolyte concentration. All of these changes were reversible, going from high to low electrolyte concentration and vice versa. In addition, it was found that the cathodic peak shifts more dramatically with $\mathrm{KCl}$ concentration than does the anodic peak, which results in asymmetrical voltammograms in dilute $\mathrm{KCl}$ solutions. The asymmetry in the shape of the cyclic voltammogram at low electrolyte concentration appears to be related to the difficulty in getting the cation into the film, since the cathodic peak shifted to a greater extent than the anodic peak.

The voltammetric behavior of the CoHNF film modified electrode in a pure electrolyte solution was also affected by the $\mathrm{pH}$ of the electrolyte solution (Fig. 4). The shape of the voltammogram was slightly changed by changing the $\mathrm{pH}$ of the solution over the range of 2 6 and the peak potentials were almost the same in this $\mathrm{pH}$ range. A further increase in the $\mathrm{pH}$ resulted in a widening of $\Delta E_{\mathrm{p}}$, and a lowering of the peak current and shifting $E_{\mathrm{m}}$ to a more anodic value. In strong alkaline media the CoHNF film was gradually disintegrated by hydrolysis. Therefore, the utility of this modified electrode is eventually limited to neutral and acidic solutions. The stability of the modified electrode was tested in a number of ways. Cycling of the potential over the range from -0.5 to $1.0 \mathrm{~V}$ was possible in numerous electrolyte solutions. For example, in a 1.0 $\mathrm{M} \mathrm{KCl}$ solution, 3000 complete cycles at $100 \mathrm{mV} / \mathrm{s}$ produced no obvious decrease in either the cathodic or anodic peak current $(<5 \%)$. Moreover, the electrode could be held at -0.5 or $1.2 \mathrm{~V}$ for $2 \mathrm{~h}$ with no observable decreases in the peak current on cyclic voltammetric scans afterwards. The CoHNF film appeared to possess good mechanical stability; when rotating the electrode or stirring the solution, only a small effect on the electrochemical stability of the modified electrode was observed. It therefore offers a very good possibili-

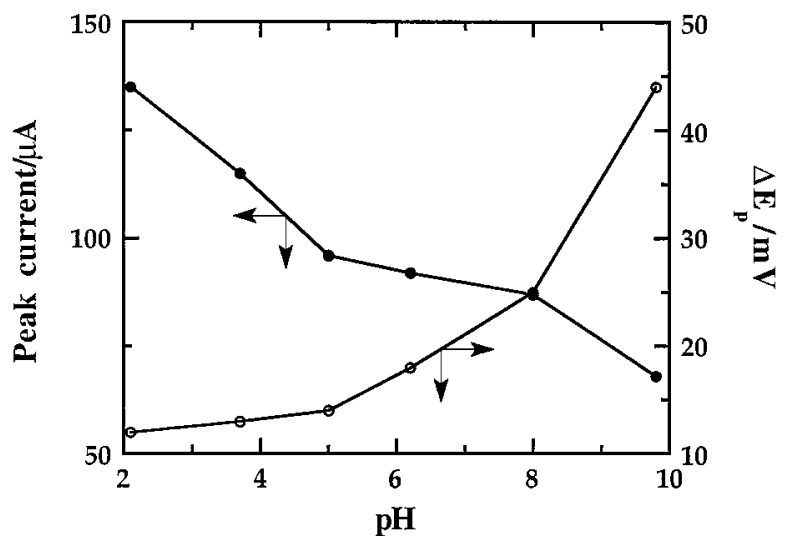

Fig. 4 Effect of $\mathrm{pH}$ on $\Delta E_{\mathrm{p}}$ and $i_{\mathrm{p}}$. The potential scan rate is $100 \mathrm{mV} / \mathrm{s}$. ty for further exploring practical applications.

Figure 5 illustrates the effect of a solvent on the cyclic voltammetry of the modified electrode. In organic solvents $\Delta E_{\mathrm{p}}$ became larger, and peak currents and stability of the film decreased greatly. The pronounced tailing of the $i-E$ curve in organic solvents indicates that the electrochemical reaction takes place rather slowly within the film. ${ }^{16}$ Also, the peak currents and potential scan-rate dependence of the film were sensitive to the solvent. Generally, in organic solvents, it seemed that these dependences are linear with the square root of scan rate, what is expected for a diffusion controlled process in spite of the film thickness. It is obvious that water is most suitable for undertaking the electrochemical redox process of the film.

\section{Spectroscopic characterization of the CoHNF films}

In order to define the surface composition of the film, we applied XPS to study an electrodeposited film on platinum foils (Table 1). When a $\mathrm{CoCl}_{2}$ solution was mixed with a $\mathrm{K}_{2}\left[\mathrm{Fe}(\mathrm{CN})_{5} \mathrm{NO}\right]$ solution, the precipitated CoHNF was contaminated by electrolyte species. The XPS spectrum of a well-washed and dried sample showed the presence of considerable amounts of $\mathrm{K}^{+}$and $\mathrm{Co}^{2+}$ ions. A survey XPS of the sample also revealed the presence the $\mathrm{Cl}^{-}$ion. However, when the CoHNF film on the platinum foil was conditioned at -0.2 and $1.0 \mathrm{~V}$ in the $1.0 \mathrm{M} \mathrm{KCl}$ solution, respectively, no observable $\mathrm{Cl}^{-}$peak was obtained in the spectra of either state of the film. As indicated in Table 1, the $\mathrm{Co} / \mathrm{Fe}$ and $\mathrm{K} / \mathrm{Fe}$ elemental ratios, as calculated from the integrated XPS high-resolution band, after a cross-sec-

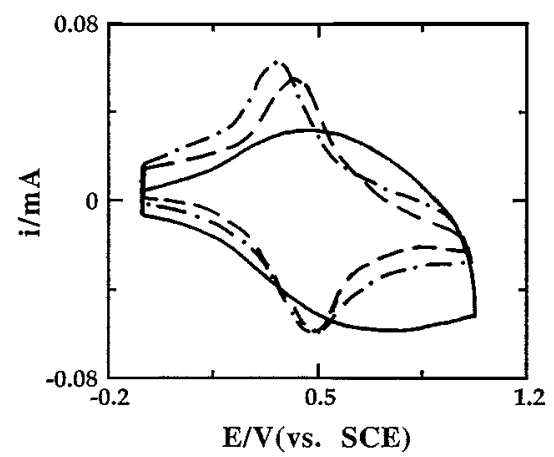

Fig. 5 Cyclic voltammograms of the modified electrode in acetonitrile ( - ), ethanol (-.-) and acetone (...) containing $0.2 \mathrm{M} \mathrm{NaClO}_{4}$. The potential scan rate is $100 \mathrm{mV} / \mathrm{s}$.

Table 1 XPS results of CoHNF films and CoHNF compound (Film 1 and 2 were obtained by electrolyzing at the respective potentials indicated)

\begin{tabular}{lcrcc}
\hline \multicolumn{1}{c}{ Sample } & $\mathrm{Co} / \mathrm{Fe}$ & \multicolumn{1}{c}{$\mathrm{K} / \mathrm{Fe}$} & $\mathrm{N} / \mathrm{Fe}$ & $\mathrm{Cl} / \mathrm{Fe}$ \\
\hline Film at $0.0 \mathrm{~V}$ & $1.4 \pm 0.5$ & $1.6 \pm 0.7$ & $8.5 \pm 1.0$ & $<0.3$ \\
Film at $1.0 \mathrm{~V}$ & $1.5 \pm 0.6$ & $<0.3 \pm 0.2$ & $7.8 \pm 1.0$ & $<0.2$ \\
$\mathrm{Co}\left[\mathrm{Fe}(\mathrm{CN})_{5} \mathrm{NO}\right]$ & $3.0 \pm 1.4$ & $8.3 \pm 1.2$ & $8.1 \pm 2.0$ & $2.2 \pm 0.8$ \\
\hline
\end{tabular}


tion correction were $1.4 \pm 0.5$ and $1.6 \pm 0.7,1.5 \pm 0.6$ and $<0.3 \pm 0.2$ for films electrolyzed at -0.2 and $1.0 \mathrm{~V}$, respectively. The detection of significant amounts of $\mathrm{K}^{+}$in the reduced film as well as the electrochemical behavior implies a $\mathrm{K}^{+}$dependence, indicating that $\mathrm{K}^{+}$is one of the components of the film. The high-resolution spectra of $\mathrm{Fe} 2 \mathrm{p}_{3 / 2}$ revealed a much broader and unsymmetrical peak with a full width at half-maximum of about $3.0 \mathrm{eV}$. This result is in good agreement with that observed in $\mathrm{Cu}\left[\mathrm{Fe}(\mathrm{CN})_{5} \mathrm{NO}\right]$ films; ${ }^{9}$ a narrow symmetric peak was observed for the ferro-species, and a broad unsymmetric peak for the ferric-compounds. ${ }^{14}$ The change in the XPS spectra of the $\mathrm{Fe} 2 \mathrm{p}_{3 / 2}$ region, sustained by the Moessbauer spectra ${ }^{19}$, establishes a redox couple at $0.51 \mathrm{~V}$ as the $\mathrm{Fe}(\mathrm{II}) / \mathrm{Fe}$ (III) couple. Moreover, FT-IR experiments revealed identical spectra for both a chemically synthesized CoHNF compound and an electrochemically deposited CoHNF film, suggesting that the deposited film is indeed made of the CoHNF compound.

Figure 6 shows an EIS plot for the modified electrode. In the high-frequency range a depressed semicircle appears, followed by a typical diffusion-controlled response; finally, the impedance approaches a purely capacitive response to account for the limiting chargesaturation effect. ${ }^{16}$ The experimental results obtained with the modified electrode may be discussed on the basis of a modified Randles equivalent circuit, taking into account the following basic aspects revealed by the voltammetric studies: (1) the film is porous in nature; (2) the kinetics of the electrode process may be controlled by diffusion of the charge-compensating ion to and from the film; and (3) the films are sufficiently thin to induce a capacitative finite length effect at low frequencies $\left(C_{\mathrm{L}}\right)$ of relevant important. When thin porous film electrodes were used, such as the CoHNF films

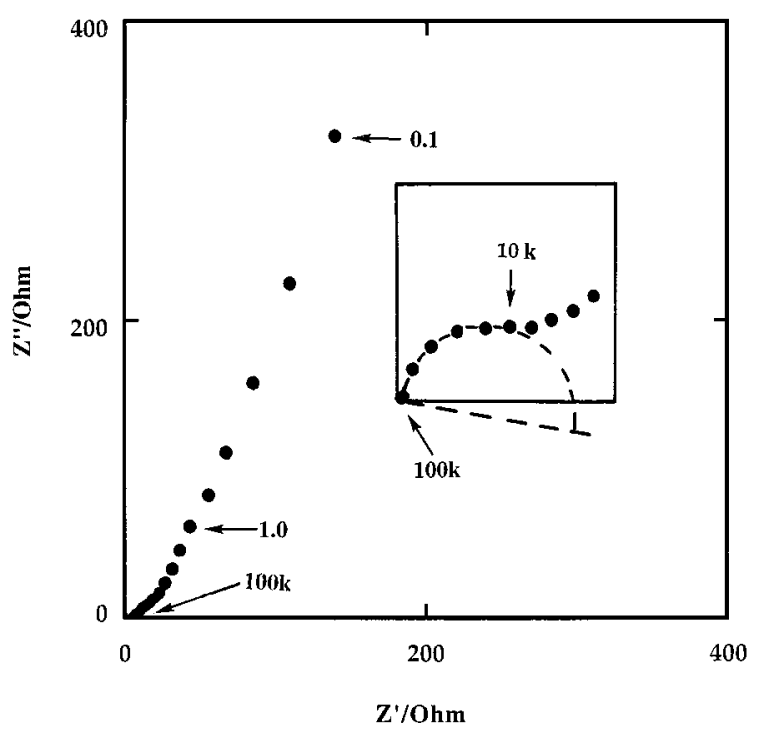

Fig. 6 EIS plot of the CoHNF-modified electrode at $0.30 \mathrm{~V}$. Insert: high frequency response. The frequencies are in $\mathrm{Hz}$.

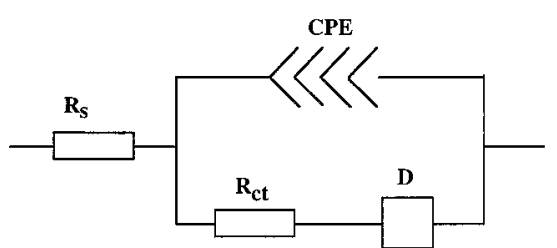

Scheme 1

studied here, the Randles circuit was modified by replacing the double-layer capacitance and the Warburg impedance with a constant-phase element (CPE) and a diffusion element $\left(Z_{\mathrm{D}}\right)$, respectively (Scheme 1$)$. The CPE is used to describe the impedance response of a porous electrode: ${ }^{16}$

$$
Z_{\mathrm{CPE}}=1 /\left[T(i \omega)^{\varphi}\right]
$$

where $T$ is a real frequency-independent constant, $i=(-1)^{1 / 2}$ and $\omega$ is the frequency in $\mathrm{rad} / \mathrm{s}$. The exponent $(\varphi)$ determines the phase angle. In the special case of $\varphi=1$, the CPE acts as a capacitor, where $T$ equals the capacitance. In the case of the CoHNF film, $\varphi$ was found to be in the range of $0.83-0.90$. $Z_{\mathrm{D}}$ is a diffusion element, which represents the finite-length diffusion process in the film. $R_{\mathrm{s}}$ and $R_{\mathrm{ct}}$ are the ohmic drop and charge-transfer resistance of the system, respectively. Under these considerations, the circuit parameters of our system were extracted from the experimental data and fitted by means of a CNLS program as previously described. ${ }^{17}$ EIS data obtained in this way are presented in Table 2.

It was found that the value of $R_{\mathrm{S}}$ neither depends on the potential nor on the film thickness. This is easily to understand by assuming that the solution resistance is associated with the electrolyte between the modified electrode and the counter electrode, and the good conductance of this kind of film. From the fitting data, the double-layer capacitance can not be directly deduced because the model with the CPE element is used; instead, $T$ is used to describe the double-layer characteristics. ${ }^{18}$ This value does not correspond to the actual capacitance, but gives a good comparison between different experiments. As shown in Table 2, $T$ was almost constant for all films, and the values are not much higher than the typical values of the electrode/electrolyte

Table 2 EIS data of the CoHNF film modified electrode at $E_{\mathrm{m}}$

\begin{tabular}{cccccc}
\hline $\begin{array}{c}\text { Film } \\
\text { thickness } / \mathrm{nm}\end{array}$ & $R_{\mathrm{S}} / \Omega$ & $R_{\mathrm{c}} / \Omega$ & $T / \mu \mathrm{s}^{\varphi} \Omega^{-1}$ & $C_{\mathrm{L}} / \mathrm{mF}$ & $10^{9} D / \mathrm{cm}^{2} \mathrm{~s}^{-1}$ \\
\hline 22 & 7.3 & 0.39 & 5.6 & 0.25 & 10.5 \\
58 & 7.4 & 0.35 & 5.9 & 0.48 & 11.6 \\
106 & 7.6 & 0.32 & 6.1 & 1.44 & 8.5 \\
160 & 7.5 & 0.30 & 6.2 & 2.08 & 10.0 \\
248 & 7.4 & 0.28 & 6.3 & 3.58 & 8.9 \\
\hline
\end{tabular}


double-layer capacitance. According to the classical double-layer model, the capacitance is independent of the potential and the film thickness, because it is only associated with the phase boundary of the electrode.

As can be seen in Table 2, a slight decrease in $R_{\mathrm{ct}}$ with the film thickness was observed, which may have been due to an increasing in the surface area of the film with increasing film thickness. On the other hand, a nearly linear relationship between $C_{\mathrm{L}}$ and the film thickness at a constant electrode area was obtained. This behavior emphasizes the insertion characteristic of the CoHNF film, since it agrees well with the theory of an insertion electrode ${ }^{19}$, which has been used to interpret the cyclic voltammetric behavior of this kind of modified electrode. ${ }^{17}$ Thus, the $\mathrm{Fe}(\mathrm{II}) / \mathrm{Fe}(\mathrm{III})$ redox centers can be considered to be elements of a capacitor, increasing with the total amount of modifier on the electrode surface, since the number of redox centers is directly proportional to the film thickness on a fixed substrate electrode area. The electrochemical impedance responses were also studied with respect to cations in the electrolyte solutions. With increasing the radius of the cation, both the charge-transfer and diffusion processes increased, which is an important feature of a chargecompensating ion controlled electrode process. The diffusion coefficient $(D)$ of the counterion across the CoHNF film was estimated from the fitting data. ${ }^{16}$ It was found that the values of $D$ were practically independent of the film thickness, suggesting that charge compensation during the redox process of the film is only associated with the ion-transport process across the film-solution interface.

\section{Electrocatalytic function of the modified electrode}

Cyclic voltammograms for the reduction and the oxidation of iron at a glassy carbon electrode and the modified electrode are shown in Fig. 7. Upon the addition of iron to the electrolyte, the oxidation current, which

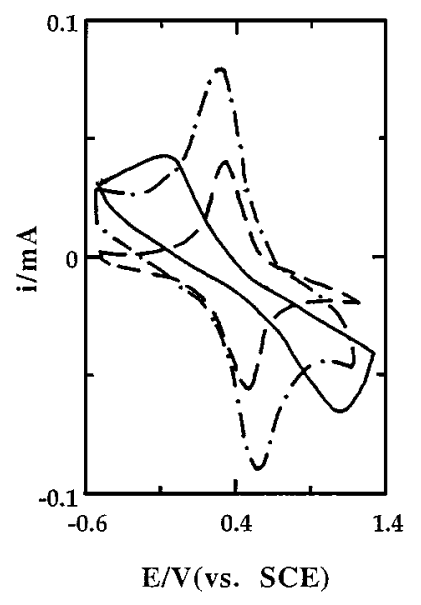

Fig. 7 Cyclic voltammograms of $1.0 \mathrm{mM} \mathrm{FeCl}_{3}+0.1 \mathrm{M} \mathrm{KCl}$ at a bare glassy carbon (-), a CoHNF modified electrode (-.-), and a modified electrode in $0.1 \mathrm{M} \mathrm{KCl}$ solution ( ...). The potential scan rate is $50 \mathrm{mV} / \mathrm{s}$. increased with increasing iron concentration, was observed. The peak potential was not affected by the iron concentration. The curve was well defined and was superimposed on the oxidation curve of the CoHNF film. This is indicative of mediated iron oxidation by the electroactive CoHNF compound. The overpotential for the oxidation of iron was lowered by about $500 \mathrm{mV}$. Over the potential scan rate range of 5 to 200 $\mathrm{mV} / \mathrm{s}$, the peak current of the catalytic oxidation of iron was found to be linear with the square root of the potential scan rate. This indicates that the electrocatalytic process is controlled by diffusion of the iron ion in the bulk solution, rather than penetration into the film, or the kinetics of the cross-exchange reactions. The above-mentioned experimental results allow two predictions. Firstly, because of the diffusion-controlled catalytic oxidation current of iron, a linear calibration curve is expected. Secondly, since the CoHNF film is highly conductive and possesses a sufficient amount of mediator on its surface to obviate the need for analyte penetration, the slope of the calibration curve should be independent of the film thickness. By employing a linear scan voltammetric mode, a calibration curve was obtained at a scan rate of $50 \mathrm{mV} / \mathrm{s}$, with an iron concentration range of $1 \times 10^{-5}$ to $8 \times 10^{-3} \mathrm{M}$ and a slope of 39.8 $\mu \mathrm{A} / \mathrm{mM}$. With a film that was $150 \%$ thicker, the slope was essentially the same. However, for the determination of lower concentration of iron, a thinner film is better because oxidation of the film, itself, occurs at the same potential as that of iron. With a thick film, it is very difficult to detect a small change in the oxidation current. The reduction of iron was also catalyzed by the CoHNF film. The overpotential was lower by about $400 \mathrm{mV}$, and the overall process was also controlled by the diffusion of iron. It therefore provides another way to detect iron in solution.

\section{Potentiometric response of a modified electrode}

In a $0.2 \mathrm{M} \mathrm{HCl}-\mathrm{KCl}(\mathrm{pH} 2.0)$ buffer solution, the CoHNF modified electrode exhibited a good potentiometric response towards $\mathrm{NO}_{2}^{-}$. However, with increasing $\mathrm{pH}$ of the medium, the response gradually deteriorated, and in an alkaline medium no detectable response was observed. Moreover, substantial decreases in voltammetric activity were observed after soaking in the alkaline medium. Figure 8 shows the potentiometric response of the modified electrode in a $\mathrm{NO}_{2}{ }^{-}$solution. The curve was representative of 6 individual electrodes which were simultaneously calibrated. The response to $\mathrm{NO}_{2}{ }^{-}$was linear over the concentration range of $3.0 \times 10^{-5}$ to $1 \times 10^{-2} \mathrm{M}$. The maximum slope was $56 \mathrm{mV}$ per decade, which is slightly smaller than the theoretical slope of $59 \mathrm{mV}$ per decade for $\mathrm{NO}_{2}{ }^{-}$. The slope of the response varied from 51 to 56 $\mathrm{mV}$ per decade. The drift of the measured e.m.f. was less than $5 \mathrm{mV} / \mathrm{h}$ in a $1.0 \mathrm{mM} \mathrm{NO}_{2}^{-}$solution. This variation is mainly due to temperature changes, electrical current fluctuations, and thermal gradients that generate potentials because of seeback effects; $;{ }^{20} 95 \%$ of the final 


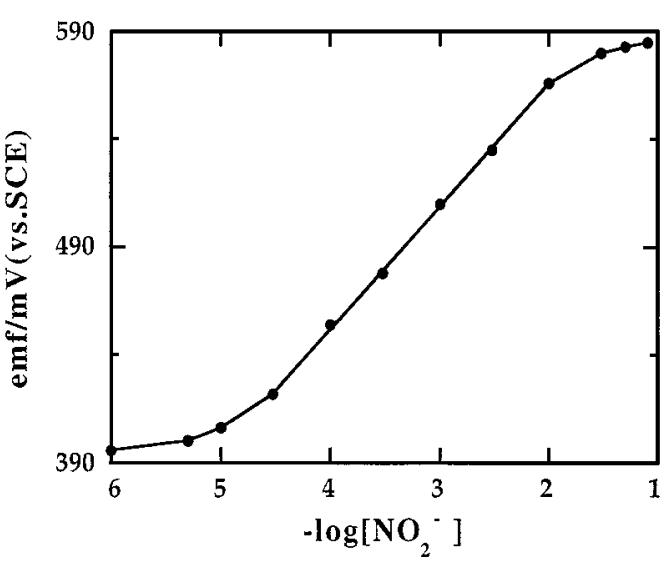

Fig. 8 Potentiometric response of the CoHNF modified electrode towards $\mathrm{NO}_{2}^{-}$in a $0.2 \mathrm{M} \mathrm{pH} 2 \mathrm{HCl}-\mathrm{KCl}$ buffer solution.

readings was achieved within 2 min. The evaluation of the detection limit for $\mathrm{NO}_{2}^{-}$, as defined by conventional IUPAC recommendation ${ }^{21}$, was $6 \times 10^{-6} \mathrm{M}$. The reproducibility of the electrode was tested in a $0.1 \mathrm{mM} \mathrm{NO}_{2}^{-}$ solution. The standard deviation for 20 parallel measurements was found to be $3.6 \%$. Detailed studies concerning the mechanism of the response and the characteristics of the electrode are underway.

In summary, the experimental results reported herein unambiguously demonstrate the feasibility of modifying a glassy carbon electrode with a novel inorganic film, CoHNF. The overall electrochemical response of the modified electrode can be interpreted by the insertion electrode model. It has also been demonstrated that the modified electrode catalyzes the oxidation and reduction of iron ions in solution. Moreover, in an acidic medium a good potentiometric response of $\mathrm{NO}_{2}{ }^{-}$ was observed at the modified electrode with a detection limit of $6 \times 10^{-6} \mathrm{M}$.

\section{References}

1. P. K. Ghosh and A. J. Bard, J. Am. Chem. Soc., 105, 5691 (1983).

2. C. G. Murry, R. J. Nowak and D. R. Rolison, J. Electroanal. Chem., 164, 205 (1984).

3. P. J. Kulesza and L. R. Faulkner, J. Am. Chem. Soc., 110, 4905 (1988).

4. M. A. Ruiz, M. G. Blazquez and J. M. Pingarron, Anal. Chim. Acta, 305, 49 (1995)

5. S. R. Snyder and H. S. White, J. Phys. Chem., 99, 5626 (1995).

6. W. Oungpipat, P. Southwell-Keely and P. W. Alexander, Analyst [London], 120, 1559 (1995).

7. B. Keita and L. Nadjo, J. Electroanal. Chem., 269, 47 (1989).

8. K. Itaya, I. Uchida and V. D. Neff, Acc. Chem. Res., 19, 162 (1986).

9. Z. Gao, Y. Zhang, M. Tian and Z. Zhao, J. Electroanal. Chem., 358, 161 (1993).

10. Z. Gao and A. Ivaska, Electrochim. Acta, 38, 379 (1993).

11. D. Ellis, M. Eckhoff and V. D. Neff, J. Phys. Chem., 85, 122 (1981)

12. A. G. Sharpe, "The Chemistry of Cyano Complexes of the Transition Metals", Academic Press, New York, 1976.

13. E. Laviron, L. Roullier and C. Degrand, J. Electroanal. Chem., 112, 11 (1980).

14. G. K. Wertheim and A. Rosenzwaig, J. Chem. Phys., 54, 3235 (1971).

15. Z. Gao, Ph.D. Dissertation, Wuhan Univ., China, 1990.

16. C. Ho, I. D. Raistrick and R. A. Huggins, J. Electrochem. Soc., 127, 343 (1980).

17. Z. Gao, J. Electroanal. Chem., 370, 95 (1994).

18. D. C. Grahame, J. Electrochem. Soc., 99, 370C (1952).

19. J. O'M. Bockris and B. Conway (ed.), "Mordern Aspects of Electrochemistry", Vol. 15, Plenum, New York, 1983.

20. D. T. Sawyer and J. L. Roberts, "Experimental Electrochemistry for Chemists", Wiley, New York, 1974.

21. IUPAC Analytical Chemistry Division, Pure Appl. Chem., 48, 127 (1976). 